\title{
ELLIPTICALLY EMBEDDED SUBGROUPS AND NILPOTENT PRODUCTS
}

\author{
by HOWARD SMITH
}

(Received 12 August, 1988)

1. Introduction. A recent paper of Rhemtulla and Wilson [4] is concerned with elliptically embedded subgroups of groups. A subgroup $H$ of a group $G$ is elliptically embedded in $G$ if, for each subgroup $K$ of $G$, there is some integer $m$ such that $\langle H, K\rangle=(H K)^{m}$. Some sufficient conditions for elliptic embedding are given in Section 2 of [4], and some consequences of the presence of this property are to be found in Theorems 1 and 2 of the same paper and in the main theorem of [5]. It is evident from all of these results that the property of being elliptically embedded is closely related to the nilpotency and subnormality of certain subgroups. One of the questions considered here is the following.

If $H, K$ are subnormal subgroups of the group $G$, when is the subgroup $J=\langle H, K\rangle$ of the form $J=(H K)^{m}$ for some integer $m$ ?

Some further conditions are certainly necessary here, even if $J$ is nilpotent (of class two)-this is indicated by the example in Section 2 of [4]. One might expect that finiteness of rank would be fairly decisive in this context, and Theorem 2.5 states, in effect, that most of the conditions known to be sufficient for $J$ to be subnormal in any group $G$ in which $H$ and $K$ are subnormally embedded also imply that $J=(H K)^{m}$ for some $m$. However, there is one such condition, concerning the tensor product of $H$ and $K$, which does not carry this implication, and a suitable example is given in Section 3. It is clear that $J=(H K)^{m+1}$ if $[H, K] \subseteq(H K)^{m}$, and we shall see that if $[H, K]$ has finite rank then there does exist such an integer $m$.

Consideration of tensor products and the subgroup $[H, K]$ leads one quite naturally to examine "nilpotent products" of $H$ and $K$. It is well known (see page 125, Vol. 1 of [7]) that $H / H^{\prime} \otimes K / K^{\prime}$ is isomorphic to [H, $\left.K\right]$ in the second nilpotent product of $H$ and $K$, and in Section 4 it is shown that this result can be partially extended to higher nilpotent products. Associated results on tensor products are also included in that section. Perhaps the main results presented here can be summarized as follows.

Let $H, K$ be groups such that $H / H^{\prime} \otimes K / K^{\prime}$ has finite rank. Then, in any nilpotent product of $H$ and $K$, the subgroup $[H, K]$ also has finite rank. Further, if $H$ and $K$ are subnormally embedded in any group $G$, then there exists an integer $m$ such that $\langle H, K\rangle=(H K)^{m}$.

2. Conditions for a union of subnormal subgroups to be an elliptic set. We begin with a lemma which will reduce the proof of the subsequent theorem to that of the "finitely generated case".

LEMMA 2.1. Let $J$ be a finitely generated nilpotent group, generated by subgroups $H$ and $K$, and suppose $[H, K]$ has rank $r$. Then $[H, K]$ is generated by $2 r$ elements of the form $[h, k]$, where $h \in H, k \in K$.

Glasgow Math. J. 31 (1989) 371-381. 
Proof. It suffices to prove that $[H, K]$ can be thus generated modulo $[H, K]^{\prime}$, since $[H, K]$ is nilpotent. Since $[H, K]^{\prime}$ is normal in $J$, we may factor and thus assume that $[H, K]$ is abelian. Let $T$ be the torsion subgroup of $[H, K]$. Then, modulo $T,[H, K]$ is free abelian of rank at most $r$, and so there is a subgroup $S$ of $[H, K]$, generated by $r$ elements of the form $[h, k]$, such that $|[H, K]: S T|$ is finite and hence $|[H, K]: S|$ is finite. Writing $S_{0}$ for the core of $S$ in $J$, we have that $\left|[H, K]: S_{0}\right|$ is finite. Modulo $S_{0}$, we need to show that $[H, K]$ is generated by $r$ elements $[h, k]$. So we assume that $[H, K]$ is finite. Then, since $J$ is residually finite, there is a normal subgroup $N$ of finite index in $J$ such that $N \cap[H, K]=1$, and we can make the further assumption that $N=1$. But $J$ is then a finite nilpotent group, and so $[H, K]$ is the direct product of subgroups $\left[H_{p}, K_{p}\right]$, where, for each prime $p$ dividing $|J|, H_{p}$ and $K_{p}$ are the Sylow $p$-subgroups of $H$ and $K$ respectively. Now each $\left[H_{p}, K_{p}\right]$ is a finite $p$-group of rank at most $r$ and so, by Burnside's Basis Theorem, can be generated by $r$ commutators of the form $\left[h_{p}, k_{p}\right]$. Thus $[H, K]$ is generated by $r$ subgroups, each of which is generated by elements $\left[h_{p_{1}}, k_{p_{1}}\right], \ldots,\left[h_{p_{n}}, k_{p_{n}}\right]$, for different primes $p_{i}$. If $\Pi_{1}, \Pi_{2}$ are disjoint sets of primes and $x_{i}, y_{i}$ are $\pi_{i}$-elements of $J(i=1,2)$ then $\left[x_{1} x_{2}, y_{1} y_{2}\right]=\left[x_{1}, y_{1}\right]\left[x_{2}, y_{2}\right]$, so by induction on the number of primes dividing $|J|$ we obtain the desired result.

In view of the fact that the nilpotency class of $J$ plays no part in the proof of the lemma above, we have the following easy consequence.

COROLlARY 2.2. Let $J$ be a locally nilpotent group, generated by subgroups $H$ and $K$, such that $[H, K]$ has finite rank $r$. Then, for each finitely generated subgroup $F$ of $[H, K]$, there exist $2 r$ commutators of the form $[h, k]$ which generate a subgroup containing $F$.

We use Lemma 2.1 to prove the following result.

THEOREM 2.3. Let $J$ be a nilpotent group of class $c$, generated by subgroups $H$ and $K$, and suppose $[H, K]$ has (finite) rank $r$. Then there is an integer $m$, depending only on $c$ and $r$, such that $J=(H K)^{m}$.

Proof. Since every element of $J$ is contained in some finitely generated subgroup of the form $\left\langle H_{0}, K_{0}\right\rangle$, where $\left[H_{0}, K_{0}\right]$ has rank at most $r$, we may suppose that $J$ is finitely generated. Let $d$ be the least integer such that $\left[H, K,{ }_{d} J\right]=1$. Then $d \leq c-1$. If $d=0$, then $J=H K$, so suppose $d \geq 1$. By Lemma $2.1,[H, K]$ is generated by elements $\left[h_{i}, k_{i}\right]$, $i=1, \ldots, 2 r$, and so, for each element $\sigma$ of $[H, K]$, there are integers $\alpha_{1}, \ldots, \alpha_{2 r}$ such that, modulo $[H, K, J]$,

$$
\begin{aligned}
\sigma & \equiv\left[h_{1}, k_{1}\right]^{\alpha_{1}} \ldots\left[h_{2 r}, k_{2 r}\right]^{\alpha_{2 r}} \\
& \equiv\left[h_{1}, k_{1}^{\alpha_{1}}\right] \ldots\left[h_{2 r}, k_{2 r}^{\alpha_{2 r}}\right] \in(H K)^{4 r} .
\end{aligned}
$$

If $d=1$, then $J=H K[H, K]=(H K)^{4 r+1}$ and we are done. Otherwise, we note that, modulo $\left[H, K,{ }_{2} J\right],[H, K, J]$ is generated by $[H, K, H]$ and $[H, K, K]$, and we apply Lemma 2.1 again to deduce that $[H, K, H]$ is generated by $2 r$ elements $\left[\sigma_{i}, g_{i}\right]$, where $\sigma_{i} \in[H, K]$ and $g_{i} \in H$ for each $i$. Then, again modulo $\left[H, K,{ }_{2} J\right]$, any element $\lambda$ of 
$[H, K, H]$ is of the form

$$
\begin{aligned}
\lambda & \equiv\left[\sigma_{1}, g_{1}\right]^{\beta_{1}} \ldots\left[\sigma_{2 r}, g_{2 r}\right]^{\beta_{2 r}} \\
& \equiv\left[\sigma_{1}, g_{1}^{\beta_{1}}\right] \ldots\left[\sigma_{2 r}, g_{2 r}^{\beta_{2 r}}\right]
\end{aligned}
$$

and each $\left[\sigma_{i}, g_{i}^{\beta_{i}}\right]$ is congruent to a product of $2 r$ elements $\left[h_{i, j}, k_{i, j}, g_{i}^{\beta_{i}}\right](j=1, \ldots, 2 r)$, each of which belongs to $(H K)^{6}$. Thus there is an element $\lambda^{\prime}$ of $(H K)^{24 r^{2}}$ such that $\lambda \equiv \lambda^{\prime}$. A similar argument applies to the subgroup $[H, K, K]$.

Then, modulo $\left[H, K,{ }_{2} J\right]$, we have $[H, K, J] \subseteq(H K)^{48 r^{2}}$. If $d=2$, we are done, otherwise we continue to consider subgroups of the $J$-central factors of $[H, K]$ in this manner and thus obtain the result required.

COROLlary 2.4. Let $G$ be a nilpotent group of class $c$ and let $H$ be a subgroup of $G$ such that $[H, G]$ has rank $r$. Then $H$ is elliptically embedded in $G$. In fact, there is an integer $m=m(r, c)$ such that, given any subgroup $K$ of $G,\langle H, K\rangle=(H K)^{m}$.

Now Proposition 3 of [4] states that if $G$ is any group and $H$ is a subgroup with the property that $H^{G}$ is nilpotent minimax, then $H$ is elliptically embedded in $G$. An obvious question is whether "minimax" could be replaced by "finite rank" here. An (unpublished) example due to Brian Hartley shows that this is not the case (see Example 3.1 below). Next, we establish some sufficient conditions for the union of two subnormal subgroups to constitute an "elliptic set", that is, we prove the following result.

THEOREM 2.5. Let $H, K$ be subnormal subgroups of the group $J=\langle H, K\rangle$. Then there is an integer $m$ such that $J=(H K)^{m}$ if any one of the following conditions is satisfied.

(i) $J^{\prime} / \gamma_{3}(J)$ has finite rank.

(ii) $[H, K]$ has finite rank modulo each term of the lower central series of $J$.

(iii) $H / H^{\prime} \otimes K / K^{\prime}$ has finite rank (where $H / H^{\prime} \otimes K / K^{\prime}$ denotes the tensor product of $H / H^{\prime}$ and $K / K^{\prime}$ viewed as abstract groups).

Proof. Each of the three conditions stated in the theorem above suffices to ensure that some term of the lower central series of $J$ is contained in the product $H K$ (see [10], [8] and [11] respectively). Hence, factoring, we may assume $J$ is nilpotent. But then $[H, K]$ has finite rank-in case (i) this follows from [9] and in case (iii) from Proposition 4.1 below. The result now follows from Theorem 2.3.

REMARK. It is easily seen (by referring to the results quoted) that the integer $m$ is in each case bounded by the subnormal defects of $H$ and $K$ and the ranks involved.

The main results of [11] are concerned with a property of abelian groups which is rather weaker than finiteness of rank, namely that of being a direct product of a group of finite rank and a periodic divisible group. Thus if $H / H^{\prime} \otimes K / K$ ' is " $F R D$ ", then the "lower central series property" (etc.) holds for $J$. However, there is no companion result for elliptic embedding, as Example 3.2 indicates. A result that we shall need to refer to in the description of this example is a consequence of the following simple observation. 
Lemma 2.6. Suppose $H, K$ are subgroups of the group $G$ and let $m$ be a positive integer. Then each element $\sigma$ of $(H K)^{m}$ can be expressed in the form

$$
\sigma=\sigma_{1} \tau_{1} \tau_{2} \sigma_{2} \ldots \sigma_{m} \tau_{m} h k
$$

where $h \in H, k \in K$ and, for each $i=1, \ldots, m, \sigma_{i}=\left[a_{1}, b_{i}\right]$ and $\tau_{i}=\left[c_{i}, d_{i}\right]$, for some $a_{i}, d_{i} \in H$ and $b_{i}, c_{i} \in K$.

Proof. If $m=1$ there is nothing to prove. Suppose $m>1$ and that $\sigma=h_{1} k_{1} \ldots h_{m} k_{m}$. Then

$$
\sigma=\left[h_{1}^{-1}, k_{1}^{-1}\right]\left[k_{1}^{-1},\left(h_{1} h_{2}\right)^{-1}\right]\left(h_{1} h_{2}\right)\left(k_{1} k_{2}\right)\left(h_{3} k_{3} \ldots h_{m} k_{m}\right),
$$

which is of the form $\sigma_{1} \tau_{1} \lambda$, where $\lambda \in(H K)^{m-1}$. The result follows by induction on $m$.

If, to the hypotheses of the lemma above, we add that $G=(H K)^{m}$ and that $H \cap K^{H}=1=K \cap H^{K}$, then it is clear that every element of $[H, K]$ is a product of at most $2 m$ commutators of the type indicated. In particular we note the following result.

Corollary 2.7. If a nilpotent product of the groups $H$ and $K$ equals $(H K)^{m}$, for some integer $m$, then every element $\sigma$ of $[H, K]$ can be expressed as a product of (at most) $2 m$ elements of the form $[h, k]$ or $[k, h]$.

We recall here that, for each positive integer $r$, the $r$-th nilpotent product of the groups $H$ and $K$ is the image of $F=H * K$ obtained by factoring by the subgroup $\left[H, K, r_{r-1} F\right]$.

3. Examples. The first example in this section is of a subgroup $H$ of a group $G$ such that $H^{G}$ is nilpotent (in fact abelian) of finite rank yet $H$ is not elliptically embedded in $G$. This is in contrast to Proposition 3 of [4] (where $H^{G}$ is nilpotent minimax) and Corollary 2.4 above (where $G$ itself is nilpotent).

EXAmPle 3.1 (Hartley). Let $k$ be a given positive integer and let $V=V_{k}$ be a maximum length $(n, k)$ code over $\operatorname{GF}(2)$, so that $n=2^{k}-1$. (See [1, p. 48] for details.) Then all the codewords have weight $d=2^{k-1}$. We claim that if $v_{1}, \ldots, v_{k-1} \in V$, then for some value of $i$, all of $v_{1}, \ldots, v_{k-1}$ have $i$-th component zero. To see this, write $S_{k}$ for a set with $2^{k}-1$ elements. We can identify each codeword with a subset of $S_{k}$. Let $\Sigma_{k}$ be the set of subsets so obtained. Then:

(i) $A_{1}, A_{2} \in \Sigma_{k}$ implies $A_{1} * A_{2} \in \Sigma_{k}$, where $*$ is the symmetric difference, and

(ii) $A \in \Sigma_{k}$ implies $|A|=2^{k-1}$ or zero.

Lemma. Conditions ( $i$ ) and (ii) imply that if $A_{1}, \ldots, A_{r} \in \Sigma_{k}$ and $A_{1} \cup \ldots \cup A_{r}=S_{k}$, then $r \geqslant k$.

Proof. If $k=2$ then $d=2, n=3$ and this is clear. Assume $k>2$ and that the result holds for smaller values and that $A_{1} \cup \ldots \cup A_{r}=S_{k}$. Let $T$ be the complement of $A_{1}$, so that $|T|=2^{k}-1-2^{k-1}=2^{k-1}-1$. Consider

$$
\Sigma^{\prime}=\left\{A \cap T: A \in \Sigma_{k}\right\} \text {. }
$$

Now if $A \in \Sigma_{k}$, then either $A=A_{1}$ and $A \cap T=\varnothing$ or $A \neq A_{1}$ and $\left|A * A_{1}\right|=2^{k-1}$. Then 2. $2^{k-1}-2\left|A \cap A_{1}\right|=2^{k-1}$, so that $2\left|A \cap A_{1}\right|=2^{k-1}$ and $\left|A \cap A_{1}\right|=2^{k-2}$. Hence 
$|A \cap T|=2^{k-1}-2^{k-2}=2^{k-2}$. Hence $D \in \Sigma^{\prime}$ implies $|D|=2^{k-2}$ or 0 ; if $D_{1}, D_{2} \in \Sigma^{\prime}$ then either $D_{1}=D_{2}$ and $D_{1} * D_{2}=\Phi$, or $D_{1} \neq D_{2}$ and $D_{i}=A_{i} \cap T$. Then

$$
D_{1} * D_{2}=\left(A_{1} * A_{2}\right) \cap T \in \Sigma^{\prime} \text {. }
$$

Thus $\Sigma^{\prime}$ satisfies the hypotheses with $k-1$ for $k$. Clearly $T=\left(A_{2} \cap T\right) \cup \ldots \cup\left(A_{r} \cap T\right)$ so that $r-1 \geqslant k-1$ and the lemma is proved.

Now writing $n=2^{k}-1$, choose $n$ distinct primes $p_{1}, \ldots, p_{n}$. Let $H_{i}, L_{i}$ be cyclic groups of order $p_{i}, M_{i}=H_{i} \times L_{i}$, and $\alpha_{i}$ an automorphism of order 2 such that $H_{i}^{\alpha_{i}}=L_{i}$. Set $N_{i}=M_{i}\left\langle\alpha_{i}\right\rangle, G_{n}=N_{1} \times \ldots \times N_{n}=M\left\langle\alpha_{1}, \ldots, \alpha_{n}\right\rangle$, where $M=M_{1} \times \ldots \times M_{n}$, and $H=\left\langle H_{1}, \ldots, H_{n}\right\rangle$. Let $K$ be the subgroup of $\left\langle\alpha_{1}, \ldots, \alpha_{n}\right\rangle$ corresponding to $V_{k}$. Now, given $i \leqslant i \leqslant n$, there exists $v \in V$ with $i$ th component $\neq 0$; this means $H^{G}=M$. But if we take any $k-1$ elements $x_{1}, \ldots, x_{k-1} \in K$, then there exists $i$ such that all of $x_{1}, \ldots, x_{k-1}$ have $i$ th component 0 , so $\left\langle H^{x_{1}}, \ldots, H^{x_{k-1}}\right\rangle \cap M_{i}=H_{i}$. Thus $L_{1} \times \ldots \times L_{n}$ is not contained in the product of any $k-1$ conjugates of $H$; hence neither is its generator, and so $L_{1} \times \ldots \times L_{n} \neq(H K)^{k-1}$.

Now write $G_{k}$ for the group above and form $G_{1} \times G_{2} \times \ldots=G$, where these are constructed from pairwise disjoint sets of primes. This contains a locally cyclic subgroup $H$ and a subgroup $K$ such that $H^{G}$ is of rank $2, K$ is elementary abelian 2-group and $G=\langle H, K\rangle \neq(H K)^{l}$ for all $l$.

Our second example concerns a (nilpotent) group $G$, generated by abelian subgroups $H$ and $K$ such that $H \otimes K$ is periodic divisible (or, rather, radicable since $G$ is written multiplicatively) but $G \neq(H K)^{m}$ for any integer $m$. This shows that the hypothesis of having finite rank in Theorem 2.5 (iii) cannot be weakened to that of being FRD (that is, a direct sum of a group of finite rank and a periodic divisible group), thus dispensing with an obvious conjecture that arises from Theorem 2.5(iii) and the main results of [11].

EXAmple 3.2. Let $p$ be a prime. For each integer $i=1,2, \ldots$, let $A_{i}$ be a group with presentation

$$
A_{i}=\left\langle a_{i 1}, a_{i 2}, \ldots: a_{i 1}^{p}=1,\left(a_{i(j+1)}\right)^{p}=a_{i j}, j=1,2, \ldots\right\rangle .
$$

Thus $A_{i}$ is a quasicyclic group of type $p^{\infty}$. Denote by $H$ the direct product of the groups $A_{i}$ and let $K$ be a free abelian group with basis $\left\{x_{1}, x_{2}, \ldots\right\}$. Then our group $G$ is defined to be the second nilpotent product of $H$ and $K$. We note that $H \otimes K$ is a divisible $p$-group and, by considering the isomorphism determined by $h \otimes k \rightarrow[h, k]$, it is clear that $\left[A_{i},\left\langle x_{j}\right\rangle\right]=\left[A_{k},\left\langle x_{l}\right\rangle\right]$ if and only if $i=k$ and $j=l$. Also, $[H, K]$ is the direct product of the subgroups $\left[A_{i},\left\langle x_{j}\right\rangle\right], i, j=1,2, \ldots$ Now let $n$ be any positive integer, and let $\sigma=\left[a_{11}, x_{1}\right] \ldots\left[a_{(n+1) 1}, x_{n+1}\right]$.

Claim. $\sigma$ cannot be written as a product of fewer than $n+1$ commutators of the form $[h, k]$, where $h \in H$ and $k \in K$.

Before we establish this claim, let us note that it has as a consequence the fact that $G \neq(H K)^{m}$ for any integer $m$. By Corollary 2.7, if $G=(H K)^{m}$, then every element of $[H, K]$ can be expressed as a product of $2 m$ commutators $[h, k]$ or $[k, h]$. But, since $G$ is 
nilpotent of class $2,[k, h]=[h, k]^{-1}=\left[h^{-1}, k\right]$ and so, putting $n=2 m$ in the above gives a contradiction.

Now assume that the claim is false and, with $\sigma$ as defined, write

$$
B=\left\langle A_{i}: i=1, \ldots, n+1\right\rangle, \quad C=\left\langle x_{i}: i=1, \ldots, n+1\right\rangle .
$$

Then, in view of our remarks concerning the structure of $[H, K]$, it follows that $\sigma$ can be written as a product of (at most) $n$ commutators of the form $[b, c]$, where $b \in B, c \in C$. Further, since each $A_{i}$ is locally cyclic, it is not difficult to see that $\sigma$ can be expressed in the form

$$
\sigma=\left[a_{1}^{\alpha_{11}} \ldots a_{n+1}^{\alpha_{(n+1) 1}}, x_{1}^{m_{11}} \ldots x_{n+1}^{m_{(n+1) !}}\right] \ldots\left[a_{1}^{\alpha_{1 n}} \ldots a_{n+1}^{\alpha_{(n+1) n}}, x_{1}^{m_{1 n}} \ldots x_{n+1}^{m_{(n+1) n}}\right]
$$

where, for each $i=1, \ldots, n+1, a_{i}$ is an element of $A_{i}$, and $\alpha_{i j}$ and $m_{i j}$ are integers $(j=1, \ldots, n)$. Further, we may assume that the order of each $a_{i}$ is the same, say $p^{k+1}$, where $k \geq 0$. So, again for $i=1, \ldots, n+1$, there is an integer $\beta_{i}\left(0<\beta_{i}<p\right)$ such that $a_{i}^{\boldsymbol{p}^{\boldsymbol{k}} \beta_{i}}=a_{i 1}$. (We could arrange for each $\beta_{i}$ to equal 1 here, but it is convenient to be less specific). From the two expressions for $\sigma$, we deduce the following system of congruences modulo $p^{k+1}$ :

$$
\begin{gathered}
S_{0, i}: \sum_{j=1}^{n} \alpha_{i j} m_{i j} \equiv \beta_{i} p^{k}, p \nmid \beta_{i}(i=1, \ldots, n+1), \\
S_{r, i}: \sum_{j=1}^{n} \alpha_{(i+r) j} m_{i j} \equiv 0, i=1, \ldots, n+1, r=1, \ldots, n,
\end{gathered}
$$

where $(i+r)$ is in each case reduced modulo $n+1$ if it is greater than $n+1$.

We show, by induction on $n$, that this system of congruences has no solution (in integers). Clearly (for a given $n$ ) we may assume that not every $\alpha_{i j}$ is divisible by $p$ (else we could divide through by $p$ and replace $k$ by $k-1$ ). By relabelling if necessary, we assume that $p$ does not divide $\alpha_{(n+1) n}$. If $n=1$, we obtain the congruences

$$
\alpha_{11} m_{11} \equiv \beta_{1} p^{k}, \quad \alpha_{21} m_{21} \equiv \beta_{2} p^{k}, \quad \alpha_{21} m_{11} \equiv 0, \quad \alpha_{11} m_{21} \equiv 0 .
$$

But the first and third of these relations imply that $\alpha_{21} \beta_{1} p^{k} \equiv 0$ and hence that $p$ divides $\alpha_{21}$, contrary to our hypothesis. Suppose then that $n \geq 2$ and that the non-existence of solutions has been established for the case $(n-1)$. Viewing the system of congruences as a system in the (integer) unknowns $m_{i j}$ (for all $i, j$ ) we note that the congruences $S_{0,1}, \ldots, S_{0, n+1}$ may be represented by the $(n+1) \times n$ coefficient matrix $P=\left(\alpha_{i j}\right)$ (augmented by the column vector $\left.p^{k}\left(\beta_{1}, \ldots, \beta_{n+1}\right)^{t}\right)$ and that, for each $r=1, \ldots, n$, the system of congruences $S_{r, 1}, \ldots, S_{r, n+1}$ is represented by a matrix $P_{r}$ obtained from $P$ by a cyclic permutation of the rows. We replace the subsystem $\left\{S_{0,1}, \ldots, S_{0, n+1}\right\}$ by the set of congruences $T_{0,1}, \ldots, T_{0, n}$ in the unknowns $m_{i j}(1 \leq i \leq n, 1 \leq j \leq n-1)$ obtained from the original system by setting $T_{0, i}=\alpha_{(n+1) n} S_{0, i}-\alpha_{i n} S_{(n+1-i) i}$; that is,

$$
\begin{aligned}
& T_{0,1}: \sum_{j=1}^{n-1}\left(\alpha_{(n+1) n} \alpha_{1 j}-\alpha_{1 n} \alpha_{(n+1) j}\right) m_{1 j} \equiv \alpha_{(n+1) n} \beta_{1} p^{k} \\
& \vdots \\
& T_{0, n}: \sum_{j=1}^{n-1}\left(\alpha_{(n+1) n} \alpha_{n j}-\alpha_{n n} \alpha_{(n+1) j}\right) m_{n j} \equiv \alpha_{(n+1) n} \beta^{n} p^{k} .
\end{aligned}
$$


Writing $Q$ for the $n \times(n-1)$ coefficient matrix appearing here and recalling that $p$ does not divide $a_{(n+1) n} \beta_{i}$ for any $i$, we see that the inductive hypothesis can be applied, provided we can deduce $(n-1)$ "homogeneous" systems of congruences in the $m_{i j}$ $(1 \leqslant i \leqslant n, 1 \leqslant j \leqslant n-1)$ whose coefficients matrices are (all) those matrices obtainable from $Q$ by cyclic permutation of the rows. But, with the aid of the following observations, it is not difficult to see that such systems can in fact be obtained by applying appropriate "row operations."

(1) For each $i=1, \ldots, n$, the $(n+1)$ st row of $P$ appears as the $i$-th row of some $P_{r}$ $(1 \leq r \leq n)$.

(2) For each pair of distinct integers $i, i^{\prime}$ in the set $\{1, \ldots, n\}$, the $i$-th row of $P$ appears as the $i^{\prime}$-th row of some $P_{r}(1 \leq r \leq n)$.

The inductive hypothesis leads to the required contradiction, so our earlier "Claim" is established, and $G \neq(H K)^{m}$ for any $m$.

4. Tensor products and nilpotent products. The following result was utilized in the proof of Theorem 2.5 .

Proposition 4.1. Suppose $H$ and $K$ are (nilpotent) groups such that the tensor product $H / H^{\prime} \otimes K / K^{\prime}$ has finite rank, and let $J$ be a nilpotent group generated by (isomorphic copies of ) $H$ and $K$. Then $[H, K]$ has finite rank in $J$.

We shall establish this proposition and some related results by considering the " $J$-central factors" of $[H, K]$ in the case where $J$ is a nilpotent product of $H$ and $K$. As remarked in the introduction, it is well known that $H / H^{\prime} \otimes K / K^{\prime}$ is isomorphic to $[H, K]$ in the second nilpotent product. The proof of the following lemma is similar to that presented in [6], and is omitted here. We denote by $\bar{X}$ the abelianisation of a group $X$, and by $\bar{x}$ the element $x X^{\prime}$.

LEMMA 4.2. Let $J$ be the $(k+2)$-nd nilpotent product of the groups $H_{1}$ and $H_{2}$, where $k$ is some non-negative integer. Then, for each $k$-tuple $\left(H_{i_{1}}, \ldots, H_{i_{k}}\right)$ of groups with $H_{i_{j}} \in\left\{H_{1}, H_{2}\right\}$ for all $j$, there is a homomorphism $\theta$ from $\bar{H}_{1} \otimes \bar{H}_{2} \otimes \bar{H}_{i_{1}} \otimes \ldots \otimes \bar{H}_{i_{k}}$ onto the subgroup $L=\left[H_{1}, H_{2}, H_{i_{1}}, \ldots, H_{i_{k}}\right]$ of $J$.

Now, with the notation of the lemma above, the subgroup $\left[H_{1}, H_{2, k} J\right]$ is generated by a finite number of subgroups having the form of $L$. We then have the following result.

Proposition 4.3. Let $\mathfrak{X}$ be a class of groups such that

(i) $X \otimes Y \in \mathfrak{X}$ implies that $X \otimes Y \otimes Y \in \mathfrak{X}$ (for any groups $X$ and $Y$ ).

(ii) Every homomorphic image of an $\mathfrak{X}$-group is in $\mathfrak{X}$.

(iii) If $G$ is an abelian group generated by the $\mathfrak{X}$-groups $A$ and $B$, then $G$ is in $\mathfrak{X}$. Let $F$ be the free product of the groups $H_{1}$ and $H_{2}$. If $\bar{H}_{1} \otimes \bar{H}_{2}$ belongs to $\mathfrak{X}$, then so does $\left[H_{1}, H_{2},{ }_{k} F\right] /\left[H_{1}, H_{2},{ }_{k+1} F\right]$ for every $k=0,1,2 \ldots$

Proof. In view of Lemma 4.2 and the subsequent remark, it need only be shown that, with the hypotheses of the Proposition satisfied, $\bar{H}_{1} \otimes \bar{H}_{2} \otimes \bar{H}_{i_{1}} \otimes \ldots \otimes \bar{H}_{i_{k}}$ also belongs to $\mathfrak{X}$ (where, as before, $H_{i_{j}}=H_{1}$ or $H_{2}$ for all $j$ ). But this is readily established by 
induction on $k$, owing to the commutativity of the tensor product operation and the presence of hypothesis (i).

Corollary 4.4. If, in addition to the hypotheses of Proposition 4.3, we also have

(iv) $\mathfrak{X}$ is closed with respect to forming extensions (that is, $\mathfrak{X}=\mathrm{P} \mathfrak{X}$ ) then $\left[H_{1}, H_{2}\right] /\left[H_{1}, H_{2}, k+1 F\right]$ is in $\mathfrak{X}(k=0,1,2, \ldots)$.

Hypothesis (iii) is of course satisfied by every $N_{0}$-closed class of groups and is implied by (ii) and (iv) above.

We now turn to the question of finding some classes $\mathfrak{X}$ which satisfy the conditions above. This involves analysis of the structure of tensor products along the lines of that carried out in Section 2 of [11]. In fact, we shall adopt the required notation from [11] as follows (see Chapter XI of [2] and Chapter X of [3] for the relevant background).

For any abelian group $A$, denote by $A_{0}$ the torsion subgroup of $A$. Let $A_{\infty}=A / A_{0}$ and let $r\left(A_{\infty}\right)$ be the rank of $A_{\infty}$. Also, for any prime $p$, let $A_{p}$ denote the $p$-component of $A_{0}$.

(1) Now let $X, Y$ be (additive) abelian groups and $T=X \otimes Y, V=T \otimes Y$. For each $p$, let $r_{p}, s_{p}, u_{p}$ be the ranks of (fixed) basic subgroups of $X_{p}, Y_{p}, T_{p}$ respectively, and let $x_{p}, y_{p}, t_{p}$ denote the ranks of $X_{\infty} / p X_{\infty}, Y_{\infty} / p Y_{\infty}, T_{\infty} / p T_{\infty}$. (Each of the ranks here is allowed to be infinite.)

With the notation as in (1), Williams [11] proves the following result.

$\left(^{*}\right) T$ is the direct sum of a group of finite rank and a periodic divisible group if and only if there is an integer $d$ such that $r\left(X_{\infty}\right) . r\left(Y_{\infty}\right), r_{p} . s_{p}, r_{p} \cdot y_{p}, x_{p} \cdot s_{p}$ and $x_{p} \cdot y_{p}$ are all at most $d$, for all primes $p$.

We use this to prove the next result.

LEMMA 4.5. If $T=X \otimes Y$ is $F R D$, then so is $T \otimes Y$.

Proof. By Lemma 2.3(ii) of [11], we have that $u_{p}=r_{p} \cdot s_{p}+x_{p} \cdot s_{p}+y_{p} \cdot r_{p}$ and so, since $T$ is FRD, $u_{p} \leq 3 d$ for some $d$ as in $\left(^{*}\right)$. Further, $r\left(T_{\infty}\right)=r\left(X_{\infty}\right) \cdot r\left(Y_{\infty}\right) \leq d$, and so $t_{p} \leq d$ also. Again by $\left(^{*}\right)$, we need only find an integer $d_{1}$ such that $r\left(T_{\infty}\right) \cdot r\left(Y_{\infty}\right), u_{p} \cdot s_{p}$, $u_{p} \cdot y_{p}, t_{p} \cdot s_{p}$ and $t_{p} \cdot y_{p}$ are all at most $d_{1}$, for all primes $p$. We deal with each of these in turn:

$r\left(T_{\infty}\right) . r\left(Y_{\infty}\right):$ If $r\left(Y_{\infty}\right)=\infty$, then $r\left(T_{\infty}\right)=0$ (since $\left.r\left(T_{\infty}\right)=r\left(X_{\infty}\right) \cdot r\left(Y_{\infty}\right) \leq d\right)$. Otherwise, $r\left(Y_{\infty}\right)=k_{1}$, say, and in either case $r\left(T_{\infty}\right) . r\left(Y_{\infty}\right) \leq d k_{1}$.

$u_{p} . s_{p}, u_{p} \cdot y_{p}$ : If $s_{p} \leq d$, then $u_{p} . s_{p} \leq 3 d^{2}$, while if $s_{p}>d$, then (by $\left.\left(^{*}\right)\right) r_{p}=0, x_{p}=0$ and so $u_{p}=0$. Similarly for $u_{p} \cdot y_{p}$.

$t_{p} \cdot s_{p}$ : If $s_{p} \leq d$, then $t_{p} \cdot s_{p} \leq d^{2}$. If $s_{p}>d$, then $x_{p}=0$, that is $X_{\infty}=p X_{\infty}$. It follows easily that $T_{\infty}=p T_{\infty}$ and so $t_{p}=0$.

$t_{p} \cdot y_{p}:$ If $y_{d} \leq d$, then $t_{p} \cdot y_{p} \leq d^{2}$, while if $y_{p}>d$, then $r\left(Y_{\infty}\right)>d$ and so $r\left(X_{\infty}\right)=0$, $r\left(T_{\infty}\right)=0$, and hence $t_{p}=0$.

Writing $d_{1}=\max \left\{d k_{1}, 3 d^{2}\right\}$, the proof of the lemma is complete.

Again, for each $p$, let $m_{p}, n_{p}$ be the ranks of the divisible components of $X_{p}, Y_{p}$ respectively. If $T$ has finite rank then there is an integer $d$ such that, in addition to the 
inequalities indicated in $\left(^{*}\right)$, we also have $y_{p} \cdot m_{p} \leq d$ and $x_{p} \cdot n_{p} \leq d$, for all $p$. Indeed, these inequalities together give a sufficient condition for $T$ to have finite rank, and (with the usual notation), we shall prove our next result.

LEMMA 4.6. If $T=X \otimes Y$ has finite rank, then $T \otimes Y$ has finite rank.

Proof. By the remarks above, it suffices to prove that $t_{p} . n_{p}$ and $y_{p}$. (the rank of the divisible component of $T_{p}$ ) are each bounded by some integer $d_{2}$ (independent of $p$ ). So we must show that $t_{p} . n_{p}, y_{p} \cdot x^{p} . n_{p}$ and $y^{2} p . m_{p}$ are bounded.

$t_{p} \cdot n_{p}:$ If $n_{p} \leq d$, then $t_{p} \cdot n_{p} \leq d^{2}$ (recall that $t_{p} \leq r\left(T_{\infty}\right) \leq d$ ). If $n_{p}>d$, then $x_{p}=0$ and (as before) $t_{p}=0$.

$y_{p}, x_{p}, n_{p}, y_{p}^{2}, m_{p}$ : If $y_{p} \leq d$, then each of these is bounded by $d^{2}$, while if $y_{p}>d$, we have $x_{p}=0=m_{p}$. The lemma is thus proved.

We can deduce some further results of this nature.

LEMMA 4.7. Condition (i) of Proposition 4.3 is satisfied by each of the following classes $\mathfrak{X}$ of groups (in addition to groups which are FRD and those that have finite rank):

(a) torsion groups, $\pi$-groups (where $\pi$ is any set of primes), groups of finite exponent,

(b) groups with the maximal or minimal condition, and minimax groups,

(c) finite groups,

(d) the class of (abelian) groups having finite $p$-ranks for all $p=0$ or a prime.

Proof. (a) is immediate, (c) is a consequence of (a) and Lemma 4.6, and (d) can be proved by examining the proofs of the previous two lemmas and noting that the relevant bounds need no longer apply for all primes at once. Abelian groups with the minimal condition are precisely those that are $\pi$-groups of finite rank for some finite set $\pi$ of primes and so (a) and Lemma 4.6 apply. If $X \otimes Y$ has the maximal condition then there are finitely generated subgroups $X_{1}$ and $Y_{1}$ of $X$ and $Y$ respectively such that $X \otimes Y \cong X_{1} \otimes Y_{1}$, and then

$$
(X \otimes Y) \otimes Y \cong\left(X_{1} \otimes Y_{1}\right) \otimes Y \cong Y_{1} \otimes\left(X_{1} \otimes Y\right) \cong Y_{1} \otimes X_{1} \otimes Y_{1},
$$

which is finitely generated.

Finally, suppose that $X \otimes Y$ is a minimax. From the structure of abelian minimax groups (see Lemma 10.31 of [7]), we infer that there are finitely generated subgroups $X_{1}$, $Y_{1}$ of $X, Y$ respectively such that, modulo $U=\left\langle x_{1} \otimes y_{1}: x_{1} \in X_{1}, y_{1} \in Y_{1}\right\rangle, X \otimes Y$ satisfies $\min$, that is, $X / X_{1} \otimes Y / Y_{1}$ satisfies $\min$ (see [2, p. 252]), as (therefore) does $Z=$ $X / X_{1} \otimes Y / Y_{1} \otimes Y / Y_{1}$. But (similarly) $Z \cong(X \otimes Y \otimes Y) / V$, where $V$ is generated by all elements $u \otimes y_{2}\left(u \in U, y_{2} \in Y_{1}\right)$. Since $V$ is a homomorphic image of $U \otimes Y_{1}$ and $U$ is an image of $X_{1} \otimes Y_{1}, V$ satisfies max. The result is now immediate.

Now it is clear that all of the classes listed in Lemma 4.7 satisfy (ii) and (iii) of Proposition 4.3 and that those given in (a), (b) and (c) are in addition P-closed. If we denote by $\mathfrak{F}_{r}^{*}$ the class of groups whose abelian sections have finite $p$-ranks for all $p=0$ or a prime, then we have established the following result. 
Lemma 4.8. The classes (a), (b), (c) of Lemma 4.7, as well as the classes $\mathfrak{F}_{r}^{*}$ and $\mathscr{\Re}_{r}$ (of groups having finite rank) satisfy the hypotheses (i)-(iv) of Proposition 4.3 and Corollary 4.4. The class FRD satisfies the first three of these hypotheses.

We remark that the class FRD is certainly not P-closed (unlike its intersection with the class of abelian groups), as may be seen from the following easy example.

Let $p$ be a fixed prime and, for each $i=1,2, \ldots$, let $A_{i}$ be as defined in Example 3.2. Let $B_{i}$ be defined similarly, and set $A=\operatorname{Dr}_{i=1}^{\infty} A_{i}, B=\operatorname{Dr}_{i=1}^{\infty} B_{i}, C=A \times B$. Define $\alpha \in$ Aut $C$ via the assignment $a_{i j} \rightarrow a_{i j} b_{i j}, b_{i j} \rightarrow b_{i j}$ for all $i, j=1,2, \ldots$ (so $\alpha^{-1}$ maps $a_{i j}$ to $a_{i j} b_{i j}^{-1}$ ). Write $\left.G=C\right]\langle\alpha\rangle$.

Then $C$ is of course divisible and $G / C$ is infinite cyclic, but $G$ is not FRD, for if $G=D \times R$, where $D$ is periodic divisible and $R$ has finite rank, we have $D$ central in $G$, and hence $G / Z(G)$ of finite rank, contradicting the fact that $A \cap Z(G)=1$.

The question as to whether the conclusion of Corollary 4.4 nevertheless holds for the class FRD is not considered here.

Proof of Proposition 4.1. Let $H, K$ be as defined. Then any nilpotent group generated by $H$ and $K$ is a homomorphic image of some nilpotent product of $H$ and $K$. The result follows from Lemma 4.8 and Corollary 4.4.

ACKNOWLEDGEMENTS. I am grateful to Akbar Rhemtulla for introducing me to some of the problems considered in this paper and for providing me with a detailed account of the group presented here as Example 3.1. Also, I thank Brian Hartley for his kind permission to include this same example in the present work.

\section{REFERENCES}

1. I. F. Blake and R. C. Mullin. The mathematical theory of coding (Academic Press, 1975).

2. L. Fuchs, Abelian groups (Pergamon Press, 1960).

3. L. Fuchs, Infinite abelian groups, Vol. 1 (Academic Press, 1970).

4. A. H. Rhemtulla and J. S. Wilson, On elliptically embedded subgroups of soluble groups, Canad J. Math., 39 (1987), 956-68.

5. A. H. Rhemtulla and J. S. Wilson, Elliptically embedded subgroups of polycyclic groups, Proc. Amer. Math. Soc. 102 (1988), 230-34.

6. D. J. S. Robinson, "A property of the lower central series of a group." Math. Z., 107 (1968), 225-231. 1972).

7. D. J. S. Robinson, Finiteness conditions and generalized soluble groups. (Springer Verlag,

8. H. Smith, Commutator subgroups of a join of subnormal subgroups, Arch. Math. (Basel), 41 (1983), 193-198.

9. H. Smith, Group-theoretic properties inherited by lower central factors, Glasgow J. Math., 29 (1987), 89-91.

10. J. P. Williams, The join of several subnormal subgroups, Math. Proc. Camb. Phil. Soc., 92 (1982), 391-399. 
11. J. P. Williams, Conditions for subnormality of a join of subnormal subgroups, Math. Proc. Camb. Phil. Soc., 92 (1982), 401-417.

Department of Mathematics

BUCKNELL UNIVERSITY

LEWISBURG

PenNSYlVANia 17837

U.S.A. 